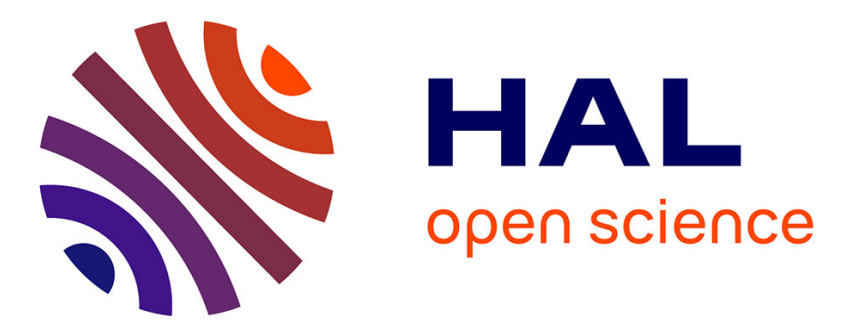

\title{
Should posterior cerebral artery occlusions be recanalized? Insights from the Trevo Registry
}

F. Clarençon, F. Baronnet, E. Shotar, V. Degos, C. Rolla-Bigliani, B.

Bartolini, E. Veznedaroglu, R. Budzik, J. English, B. Baxter, et al.

\section{- To cite this version:}

F. Clarençon, F. Baronnet, E. Shotar, V. Degos, C. Rolla-Bigliani, et al.. Should posterior cerebral artery occlusions be recanalized? Insights from the Trevo Registry. European Journal of Neurology, 2020, 27 (5), pp.787-792. 10.1111/ene.14154 . hal-02512399

\section{HAL Id: hal-02512399 \\ https://hal.sorbonne-universite.fr/hal-02512399}

Submitted on 29 Jun 2020

HAL is a multi-disciplinary open access archive for the deposit and dissemination of scientific research documents, whether they are published or not. The documents may come from teaching and research institutions in France or abroad, or from public or private research centers.
L'archive ouverte pluridisciplinaire HAL, est destinée au dépôt et à la diffusion de documents scientifiques de niveau recherche, publiés ou non, émanant des établissements d'enseignement et de recherche français ou étrangers, des laboratoires publics ou privés. 


\section{Should posterior cerebral artery occlusions be recanalized? Insights from the Trevo Registry}

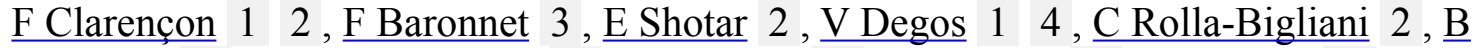
Bartolini $5, \underline{\text { E Veznedaroglu }} 6, \underline{\text { R Budzik }} 7$, $\underline{\text { English }} 8, \underline{B}$ Baxter $9, \underline{\mathrm{D} S}$

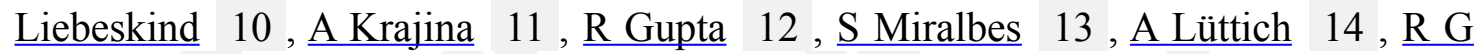

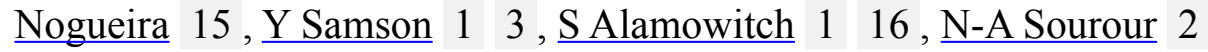

\section{Affiliations}

1 Sorbonne University, Paris, France.

2 Department of Neuroradiology, Pitié-Salpêtrière Hospital, Paris, France.

3 Department of Vascular Neurology, Pitié-Salpêtrière Hospital, Paris, France

4 Department of Anesthesiology, Pitié-Salpêtrière Hospital, Paris, France.

5 Department of Radiology, Interventional Neuroradiology Unit, Lausanne University Hospital, Lausanne, Switzerland.

6 Endovascular Neurosurgery, Drexel Neurosciences Institute, Philadelphia, PA, USA.

7 Interventional Neuroradiology, Riverside Methodist Hospital/Ohio Health Research Institute, Columbus, OH, USA.

8 Interventional Neurology, California Pacific Medical Center, San Francisco, CA, USA.

9 Interventional Neuroradiology, Erlanger, Chattanooga, TN, USA.

10 Department of Neurology and Comprehensive Stroke Center, David Geffen School of Medicine, University of California, Los Angeles, CA, USA.

11 Department of Radiology, University Hospital Hradec Kralove, Hradec Kralove, Czech Republic.

12 Interventional Neurology, Wellstar Health System, Atlanta, GA, USA.

13 Hospital Universitario Son Espases, Mallorca, España.

14 Hospital de Donostia, San Sebastian, España.

15 Department of Neurology, Marcus Stroke and Neuroscience Center/Grady Memorial Hospital/Emory University, Atlanta, GA, USA.

16 Department of Vascular Neurology, Saint Antoine University Hospital, Paris, France. 


\section{Abstract \\ Background and Aims}

The purpose of this study was to evaluate the safety and effectiveness of mechanical thrombectomy (MT) in patients with acute ischemic stroke (AIS) related to isolated and primary posterior cerebral artery (PCA) occlusions among the patients enrolled in the multicentre post-market Trevo Registry.

\section{Method}

Among the 2008 patients enrolled in the Trevo Registry with AIS due to large vessel occlusion (LVO) treated by MT, 22 (1.1\%) (10 females [45.5\%], mean age: $66.2 \pm 14.3$ years [range 28-91]) had a PCA occlusion (17 P1 [77.3\%] and five P2 occlusions [22.7\%]). Recanalization after the first Trevo (Stryker, Fremont, CA) pass and at the end of the procedure was rated using the mTICI score. Procedure-related complications (i.e.: groin puncture complication, perforation, symptomatic hemorrhage, embolus in a new territory) were also recorded. Modified Rakin Scale (mRS) at 90 days was assessed.

\section{Results}

Median NIHSS at admission was 14 [IQR, 8-16]. Stroke etiology was cardio-embolic in $68.2 \%$ of cases. Half of the patients $(11 / 22)$ received IV tPA. $54.5 \%$ of the patients were treated under general anesthesia. Reperfusion (i.e.: mTICI2b or 3) after first pass was obtained in $65 \%$ of cases. Final TICI $2 b-3$ reperfusion was obtained in all cases. Only one $(4.5 \%)$ procedure-related complication was recorded (puncture site), that resolved after surgery. At 90-day follow-up, mRS 0-2 was obtained in 59\% of the patients and $9.1 \%$ died within the first 3 months after MT.

\section{Conclusion}

Mechanical thrombectomy for PCA occlusions seem to be safe $(<5 \%$ procedurerelated complications) and effective. Larger repository datasets are needed. 


\section{Introduction}

Acute ischemic strokes (AIS) involving the posterior cerebral artery (PCA)'s territory are rare; they account for approximately $5-10 \%$ of all AIS (1-3). Most of these PCA strokes are caused by a cardio-embolic pathomechanism (4). AIS related to PCA occlusion are even rarer; they have been reported accounting for about $0.5 \%$ of all AIS with large vessel occlusion (LVO) (5). This specific subtype of AIS is usually responsible for lower NIHSS strokes (1), but with potential life altering consequences such as inability to drive due to a visual impairment or alexia. These strokes are felt to be less severe compared to basilar artery occlusion or anterior circulation strokes (4). Additionally, the clinical symptoms revealing PCA infarcts are less recognized; which could be responsible for a delayed diagnosis, out of the time-window for intravenous (IV) thrombolysis (6).

Recent randomized control trials (RCTs) have proven the effectiveness of mechanical thrombectomy (MT), combined with the best medical treatment, in AIS with LVO involving the anterior circulation (7). However, data regarding the safety and effectiveness of MT in patients with AIS related to PCA occlusion are lacking. There have been limited reports for treating this condition to date $(8,9)$.

The purpose of our study was to evaluate the safety and recanalization rates of MT in PCA occlusions. 


\section{Materials and Methods}

Study design:

Post-hoc analysis of a prospective multicentre post-market registry (the Trevo Retriever Registry; ClinicalTrials.gov identifier: NCT02040259). The Trevo Retriever Registry is a prospective, open-label, consecutive enrolment, multi-center, international registry aiming to collect real world performance data of the Trevo Retriever (Stryker, Fremont, CA) which is intended to restore blood flow in the neurovasculature by removing thrombus in patients experiencing AIS (10).

Patients'demographics/Inclusion criteria:

From November 11, 2013 to May 1, 2017, a total of 2008 patients were enrolled in the TREVO Retriever Registry (1041 females, 51.8\%; mean age: $68.3 \pm 14.4$ years with a range of 19 to 99 years).

Among the patients enrolled in the Trevo Retriever Registry, only those with DSA proven isolated and primary PCA occlusion(s), from P1 to P4 (11), were included. Location of the occlusion site was evaluated by an independent core-lab. Patients with basilar artery occlusion associated with PCA occlusion were excluded. Patients with secondary PCA occlusions (i.e.: thrombus migration in PCA during basilar artery MT) were also excluded.

For each patient retained with a PCA occlusion, demographic data, time of symptoms' onset, and past medical history (history of coronary artery disease, atrial fibrillation, previous transient ischemic attack [TIA], or ischemic stroke) were systematically 
recorded. The following cardiovascular risk factors were collected: blood hypertension, diabetes mellitus, current cigarette smoking, dyslipidemia, coronary artery disease.

\section{Patients 'management}

In all cases, the patient was examined at admission by a stroke-trained provider who graded the NIHSS (National Institutes of Health Stroke Scale). After clinical examination, all patients had a standard investigation protocol including blood tests, electrocardiography, brain computed tomography (CT) scan, and/or magnetic resonance imaging (MRI). LVO was documented noninvasively by a CT angiography (CTA) and/or a magnetic resonance angiography (MRA). Cervical vessel evaluation was performed at the discretion of the team in charge of the patient.

Patients who were admitted within the appropriate time-window, and without contraindication, received intravenous (IV) rt-PA (recombinant tissue plasminogen activator) $(0.9 \mathrm{mg} / \mathrm{kg})$ as per their local institutional protocol. Patients were treated either under general anesthesia (GA) or under conscious sedation/local anesthesia, at the discretion of the operator.

\section{Occlusion site/stroke subtype classification}

The arterial occlusion site was evaluated on DSA in anteroposterior (AP) and lateral projections and graded according to the Fischer's segmentation (from P1 to P4) (11). Stroke aetiology was classified according to the Trial of Org 10172 in Acute Stroke Treatment (TOAST) criteria (12).

\section{Safety/intracranial hemorrhage}


Procedure-related complications were divided into 1) minor complications: groin puncture minor complications that did not require blood transfusion or surgical repair, minor intracranial hemorrhage that did not lead to clinical worsening; and 2) major complications: intracranial arterial perforation, embolus in a new territory (ENT) leading to clinical worsening, symptomatic intracranial hemorrhage (i.e.: intracranial hemorrhage leading to a clinical worsening [4-point increasing on the NIHSS score]), cervical arterial dissection responsible for clinical worsening, groin puncture complications requiring blood transfusion or surgical repair.

Identification and classification of intracranial hemorrhage was performed routinely on brain MRI or CT within 24h after MT or in case of any clinical deterioration. ICH was classified as "symptomatic" if a parenchymal hematoma type 2 (according to the ECASS criteria) was accompanied by a 4-point or greater increase in the NIHSS score or if leading to death $(13,14)$.

\section{Angiographic and clinical outcomes}

Recanalization after the first Trevo pass and at the end of the procedure was rated using the mTICI score (15) on AP and lateral projections DSA by an independent core lab. Successful reperfusion was defined as mTICI $2 \mathrm{~B} / 3 . \mathrm{mRS}$ at 90 days was systematically recorded by a certified physician for mRS assessment. A mRS $\leq 2$ at 3 months' followup was considered as a good clinical outcome.

\section{Statistical analysis:}

We compiled descriptive statistics for parameters of interest for our analysis. 
Reported p-values are two-sided and are not adjusted for multiple comparisons considering the exploratory nature of the analyses. All analyses were conducted using SAS software (SAS, Version 9.4, SAS Institute Inc., Cary, NC).

\section{Ethical statement:}

The registry was approved by the institutional review board at each site. Patients or their designee provided written informed consent within 7 days of the procedure. 


\section{Results}

Out of the 2008 patients included in the Trevo Retriever registry, $22(1.1 \% ; 10$ females [45.5\%], mean age: $66.2 \pm 14.3 \mathrm{y}$ [range 28-91]) presented an AIS related to a primary and isolated PCA occlusion treated by MT with the Trevo Retriever device as first line strategy. Occlusion sites were distributed as follows: $17 \mathrm{P} 1$ occlusions [77.3\%] and 5 P2 occlusions [22.7\%]. Neither P3 nor P4 occlusions were included in our study cohort. Median NIHSS at admission was 14 (Q1-Q3: 8-16; range: 1-26). Stroke etiology was cardio-embolic in most cases $(68.2 \%)$, followed by large artery atherosclerosis $(13.6 \%)$. Half of the patients $(50 \%)$ received IV rt-PA. Interestingly, half of the patients $(11 / 22)$ underwent the MT over the 4 h30 time-window after the symptoms' onset. In $54.5 \%$ of the cases, the patients were treated under general anesthesia (Baseline patient characteristics are summarized in Additional Material \#

\section{1; Procedural characteristics are summarized in Additional Material \# 2).}

Reperfusion (i.e.: mTICI2b or 3) after first pass with the Trevo Retriever was obtained in $65 \%$ of cases (mTICI 3 in $50 \%$ of the cases). Final mTICI $2 b-3$ reperfusion was obtained in $100 \%$ of the cases. In average $1.3 \pm 0.5$ passes (range: $1-2$ ) of the Trevo Retriever were performed; $1.6 \pm 0.9$ (range: 1-4) for all thrombectomy devices (Clinical assessment and efficacy outcomes are displayed in Table 1).

Only one (3.7\%) severe procedure-related complication was recorded (puncture site), that resolved after surgery. No device (Trevo)-related complication occurred. One case (4.5\%) of clinical worsening during the $24 \mathrm{~h}$ after the MT was observed due to increased of the mass effect related to the infarct and which was not a procedurerelated complication. Additionally, no embolus migration in another territory was recorded. No vessel perforation was observed, as well. Intracranial hemorrhages were 
observed in $18.2 \%$ of the cases ( $3 \mathrm{HI} 1$ and 1 subarachnoid hemorrhage). No symptomatic hemorrhages according to the ECASS 3 study classification were recorded; asymptomatic hemorrhages were observed in $18.2 \%(4 / 22)$ of the cases.

Median NIHSS at 24h after the MT was 3 (Q1-Q3: 1-8; range: 0-27). At 90-day follow-up, 59.1\% (13/22) of the patients had a mRS $0-2 ; 9.1 \%(2 / 22)$ died within the first 3 months after the MT. 


\section{Discussion}

Acute ischemic strokes related to PCA occlusion are difficult to diagnose due to a frequent non-specific clinical presentation, which can explain the delay in their management, sometimes beyond the time-window for IV rt-PA. Since the symptoms and sequelae of AIS related to PCA occlusion are usually less severe, one may ask if MT is a suitable option for patients with PCA occlusion. Indeed, compared to middle cerebral artery (MCA) or anterior cerebral artery (ACA), sequelae after PCA occlusions are usually milder. However, P1 or P2 occlusions may involve perforating branches feeding the thalamus (16) which could lead to severe sequelae. Additionally, infarct the PCA's cortical territory may be responsible for severe hemianopia, which could be severely disabling, especially in young patients.

The aim of our study was to evaluate the safety and effectiveness of MT for AIS with PCA occlusions. Our sub-group analysis of MT for PCA occlusions from the Trevo Retriever registry showed the safety of this procedure for this specific occlusion site. Indeed, only one $(<5 \%)$ major procedure-related complication was recorded, which consisted in a groin puncture complication requiring surgical management, but which did not lead to clinical sequelae. It is noteworthy that this major complication rate is within the range of the ones reported in the literature for anterior circulation MT (17). Technically, there was a $100 \%$ successful reperfusion rate. Moreover, there were $18.2 \%$ of patients with asymptomatic hemorrhages and $81.8 \%(18 / 22)$ with improvement in their NIHSS at discharge. At 3-months follow-up, 59.1\% of the patients had a good clinical outcome $(\mathrm{mRS} \leq 2)$. All these results are within the ranges of the ones reported for comparable-size arterial occlusions (i.e.: distal occlusions) of the anterior circulation (18). 
Interestingly, admission NIHSS scores were higher in our study population (median: 14) than reported in series with patients treated by IV thrombolysis for PCA territory AIS ${ }^{21}$. However, one should keep in mind that all the patients involved in our study had a proximal (P1 or P2) PCA occlusion, which may explain this discrepancy. Only limited data are available in the literature on the safety and effectiveness of MT in PCA occlusions; mainly case reports, either of regular (8) or fetal (19) PCA configurations, and short case series in which PCA occlusions were merged with other posterior circulation occlusions (20). In a recent study comparing anterior and posterior circulation strokes (20), only $6(4.3 \%)$ isolated PCA occlusions were reported and 23 cases $(16.5 \%)$ of PCA or vertebral artery occlusions combined with basilar artery occlusion. No sub-group analysis was available in this study on PCA occlusions (either isolated or combined) (20).

A short case series (21) comparing intra-arterial (IA) thrombolysis with IV rt-PA has shown no superiority of IA over IV thrombolysis in PCA occlusions in terms of good clinical outcome (mRS $\leq 2$ in $67 \%$ of the cases for IA thrombolysis and $89 \%$ with IV thrombolysis). However, the number of patients included in this case series was very low (9 patients in each group [IA and IV]). Additionally, no randomization was performed for the technique used.

\section{Limitations}

The main limitation of our sub-group study analysis is the low volume of the patients with a PCA occlusion included. However, the Trevo Retriever Registry is a very large registry reflecting the real life daily practice in MT. The second limitation is the lack of randomization to compare MT to the best medical treatment. Indeed, PCA being a 
relatively small artery, one could assume that rt-PA may be more effective in this specific localisation, compared to ICA terminus or proximal M1. Additionally, our study lacks specific data on visual evaluation, like visual field defects, as well as on neuropsychological examination. Such more specific evaluation would have been valuable for the assessment of sequelae related to the PCA's territory AIS. Finally, only P1 to P2 occlusions were included. The good results obtained in our case series in terms of recanalization and low complication rate may probably not be extrapolated to more distal (i.e.: P3 or P4) occlusions. 


\section{Summary}

The post-hoc analysis of patients included in the Trevo Retriever Registry who underwent a MT for an isolated and primary PCA occlusion shows the safety of this technique with a low rate of major complication $(<5 \%)$. MT in PCA occlusion seems effective with a high recanalization rate (100\%). However, only a randomized controlled trial could prove the superiority of MT associated with the BMT over the BMT alone in terms of good neurological outcome for this specific subtype of acute ischemic stroke. 


\section{References}

1. Arboix A, Arbe G, Garcia-Eroles L, Oliveres M, Parra O, Massons J. Infarctions in the vascular territory of the posterior cerebral artery: clinical features in 232 patients. BMC Res Notes. 2011;4:329.

2. Kinkel WR. Vertebrobasilar Arterial Occlusive Disease. New York RP, editor1984.

3. Kleihues P, Hizawa K. [The infarct of the posterior cerebral artery: pathogenesis and topographical relationship to the visual cortex]. Arch Psychiatr Nervenkr (1970). 1966;208(3):263-84.

4. Yamamoto Y, Georgiadis AL, Chang HM, Caplan LR. Posterior cerebral artery territory infarcts in the New England Medical Center Posterior Circulation Registry. Arch Neurol. 1999;56(7):824-32.

5. Rai AT, Seldon AE, Boo S, Link PS, Domico JR, Tarabishy AR, et al. A population-based incidence of acute large vessel occlusions and thrombectomy eligible patients indicates significant potential for growth of endovascular stroke therapy in the USA. J Neurointerv Surg. 2017;9(8):722-6.

6. Sand KM, Naess H, Nilsen RM, Thomassen L, Hoff JM. Less thrombolysis in posterior circulation infarction-a necessary evil? Acta Neurol Scand. 2017;135(5): 546-52.

7. Saver JL, Goyal M, van der Lugt A, Menon BK, Majoie CB, Dippel DW, et al. Time to Treatment With Endovascular Thrombectomy and Outcomes From Ischemic Stroke: A Meta-analysis. JAMA. 2016;316(12):1279-88.

8. Yamamoto T, Ohshima T, Sato M, Goto S, Ishikawa K, Nishizawa T, et al. A Case of Acute Isolated Posterior Cerebral Artery Occlusion Successfully Treated with Endovascular Clot Aspiration. NMC Case Rep J. 2017;4(2):55-8.

9. Lee HN, Kim BT, Im SB, Hwang SC, Jeong JH, Chung MY, et al. Implications of Mechanical Endovascular Thrombectomy for Acute Basilar and Posterior Cerebral Artery Occlusion. J Cerebrovasc Endovasc Neurosurg. 2018;20(3):168-75.

10. Binning MJ, Bartolini B, Baxter B, Budzik R, English J, Gupta R, et al. Trevo 2000: Results of a Large Real-World Registry for Stent Retriever for Acute Ischemic Stroke. J Am Heart Assoc. 2018;7(24):e010867.

11. Fischer E. Die Lageabweichungen der vorderen Hirnarterie imGefäßbild. Zentralbl Neurochir. 1938;3:300-13.

12. Adams HP, Jr., Bendixen BH, Kappelle LJ, Biller J, Love BB, Gordon DL, et al. Classification of subtype of acute ischemic stroke. Definitions for use in a multicenter clinical trial. TOAST. Trial of Org 10172 in Acute Stroke Treatment. Stroke. 1993;24(1):35-41.

13. Hacke W, Kaste M, Fieschi C, von Kummer R, Davalos A, Meier D, et al. Randomised double-blind placebo-controlled trial of thrombolytic therapy with intravenous alteplase in acute ischaemic stroke (ECASS II). Second EuropeanAustralasian Acute Stroke Study Investigators. Lancet. 1998;352(9136):1245-51.

14. Wahlgren N, Ahmed N, Davalos A, Ford GA, Grond M, Hacke W, et al. Thrombolysis with alteplase for acute ischaemic stroke in the Safe Implementation of Thrombolysis in Stroke-Monitoring Study (SITS-MOST): an observational study. Lancet. 2007;369(9558):275-82. 
15. Zaidat OO, Yoo AJ, Khatri P, Tomsick TA, von Kummer R, Saver JL, et al. Recommendations on angiographic revascularization grading standards for acute ischemic stroke: a consensus statement. Stroke. 2013;44(9):2650-63.

16. Schmahmann JD. Vascular syndromes of the thalamus. Stroke. 2003;34(9): 2264-78.

17. Chivot C, Deramond H, Bouzerar R, Yzet T. Safety and Efficacy of Femoral Artery Closure with the FemoSeal Device After Cerebral Thrombectomy Using an 8 French Sheath. Eur J Vasc Endovasc Surg. 2018;55(5):730-4.

18. Chen CJ, Wang C, Buell TJ, Ding D, Raper DM, Ironside N, et al. Endovascular Mechanical Thrombectomy for Acute Middle Cerebral Artery M2 Segment Occlusion: A Systematic Review. World Neurosurg. 2017;107:684-91.

19. Amuluru K, Ho JP, Al-Mufti F, Solander S, Romero CE. Endovascular intervention of acute ischemic stroke due to occlusion of fetal posterior cerebral artery. Interv Neuroradiol. 2018:1591019918801285.

20. Weber R, Minnerup J, Nordmeyer H, Eyding J, Krogias C, Hadisurya J, et al. Thrombectomy in posterior circulation stroke: differences in procedures and outcome compared to anterior circulation stroke in the prospective multicentre REVASK registry. Eur J Neurol. 2018.

21. Meier N, Fischer U, Schroth G, Findling O, Brekenfeld C, El-Koussy M, et al. Outcome after thrombolysis for acute isolated posterior cerebral artery occlusion. Cerebrovasc Dis. 2011;32(1):79-88. 
Table 1. Clinical assessment and efficacy outcomes

ITT P1 P2 ( $\mathbf{N = 2 2 )}$

First Trevo Pass Modified TICI $\geq 2 b$

$65 \%(12 / 20)$

\section{First Trevo Pass Modified TICI}

Grade 0

$25.0 \%(5 / 20)$

Grade 1

$10.0 \%(2 / 20)$

Grade 2a

$0 \%(0 / 20)$

Grade $2 b$

$15 \%(3 / 20)$

Grade 3

$50.0 \%(10 / 20)$

Not available

$9 \%(2 / 22)$

Post-procedure Modified TICI $\geq 2 b$

$100 \%(22 / 22)$

\section{Pre-stroke mRS}

0

$85.7 \%(18 / 21)$

$4.8 \%(1 / 21)$

2

$9.5 \%(2 / 21)$

90-day mRS $0-2$

$59.1 \%(13 / 22)$

\section{0-day mRS}

0

$22.7 \%(5 / 22)$

$22.7 \%(5 / 22)$

2

$13.6 \%(3 / 22)$

3

$18.2 \%(4 / 22)$

4

$13.6 \%(3 / 22)$ 


\section{Pre-procedure NIHSS}

Mean \pm SD

Median (Q1-Q3)

Range (Min-Max)
$12.6 \pm 6.6(21)$

$14(8-16)$

$1-26$

\section{4-hour NIHSS}

Mean \pm SD

Median (Q1-Q3)

Range (Min-Max)
$6.6 \pm 7.3(26)$

$3(1-8)$

$0-27$

NIHSS at Discharge or Day 5-7, whichever is earlier

Mean \pm SD

Median (Q1-Q3)

Range (Min-Max)
$5.2 \pm 7.8(20)$

$1.5(1-7)$

$0-27$

ITT indicates intention to treat, $\mathbf{N}$ : number, TICI: thrombolysis in cerebral infarction, SD: standard deviation, Min: minimum, Max: maximum 